\title{
Strong anti-kinetoplastid activity of silver nanoparticle-coated biochar
}

\author{
Youssef Snoussi ${ }^{1}$, Inès Sifaoui ${ }^{2,3,4,5}$, Ahmed M. Khalil ${ }^{6,7}$, Arvind K. Bhakta ${ }^{1,8}$, \\ Laurent Michely ${ }^{7}$, Rémy Pires ${ }^{7}$, Stéphane Bastide ${ }^{7}$, Oleg Semyonov ${ }^{9}$, Pavel S. Postnikov ${ }^{9}$, \\ José Enrique-Piñero Barroso ${ }^{2,3,4,5}$, Jacob Lorenzo Morales ${ }^{2,3,4,5,{ }^{*}}$, Mohamed M. Chehimi ${ }^{1,7, *}$ \\ ${ }^{1}$ Université de Paris, CNRS, ITODYS (UMR 7086), 75013 Paris, France \\ 2 Instituto Universitario de Enfermedades Tropicales y Salud Pública de Canarias, \\ Universidad de La Laguna, Avda. Astrofísico Fco. Sánchez, S/N, La Laguna, Tenerife, \\ 38203, Islas Canarias, Spain \\ ${ }^{3}$ Departamento de Obstetricia, Ginecología, Pediatría, Medicina Preventiva y Salud Pública, \\ Toxicología, Medicina Legal y Forense y Parasitología, Universidad de la Laguna (ULL), La \\ Laguna, Tenerife, 38203 Islas Canarias, Spain \\ ${ }^{4}$ Red de Investigación Cooperativa en Enfermedades Tropicales (RICET), 28029 Madrid, \\ Spain \\ ${ }^{5}$ CIBER de Enfermedades Infecciosas (CIBERINFEC), Instituto de Salud Carlos III, 28029 \\ Madrid, Spain \\ ${ }^{6}$ Photochemistry Department, National Research Centre, Dokki, Giza 12622, Egypt \\ ${ }^{7}$ Université Paris Est, CNRS, ICMPE (UMR 7182), 94320 Thiais, France \\ ${ }^{8}$ Department of Chemistry, St. Joseph's College, Lalbagh Road, \\ Bangalore, Karnataka, 560 027, India \\ ${ }^{9}$ Tomsk Polytechnic University, 634050 Tomsk, Russian Federation
}

\begin{abstract}
Neglected tropical diseases including Chagas disease, also known as American trypanosomiasis and leishmaniasis remains a serious health problem in several endemic. To address this medical problem, much has been done in the past 15 years to design nanomaterials with effective anti-kinetoplastids activity, particularly those nanomaterials based on gold and silver nanoparticles. Herein, we describe a simple method to prepare silverloaded biochar by pyrolysis of silver nitrate-impregnated agrowaste powder (from olive stones). The resulting Biochar@ Ag was prepared at $400{ }^{\circ} \mathrm{C}$ for 15 minutes only and the yield was found to be $36.5 \%$. The supported metallic Ag nanoparticles have triangular shape in the nanoscale regime $(<100 \mathrm{~nm})$ and a loading of $7.85 \mathrm{mmol}$ per gram of Biochar@ Ag. The Biochar@Ag showed promising antiparasitic activity against promastigotes stage of $L$. donovani, L. amazonensis and epimastigotes of $T$. cruzi with and $\mathrm{IC}_{50}$ of $9.942 \pm 0.900 \mathrm{ppm}$; $14.555 \pm 1.035 \mathrm{ppm}$ and $12.154 \pm 0.206 \mathrm{ppm}$, respectively. From the above, this work conclusively demonstrates that slow pyrolysis is a unique thermochemical approach to valorize agrowastes into highly effective anti-kinetoplastid silver-loaded biochar with remarkably low cytotoxicity towards murine macrophages.
\end{abstract}

\section{Keywords:}

Leishmania; neglected tropical disease; antiparasitic nanocomposite; agrowaste; olive pit; biochar; silver nanoparticles; trash to treasure.

\section{Corresponding authors}

Jacob Lorenzo Morales: jmlorenz@ull.edu.es ;

Mohamed M. Chehimi: mohamed.chehimi@cnrs.fr 


\section{Graphical Abstract}

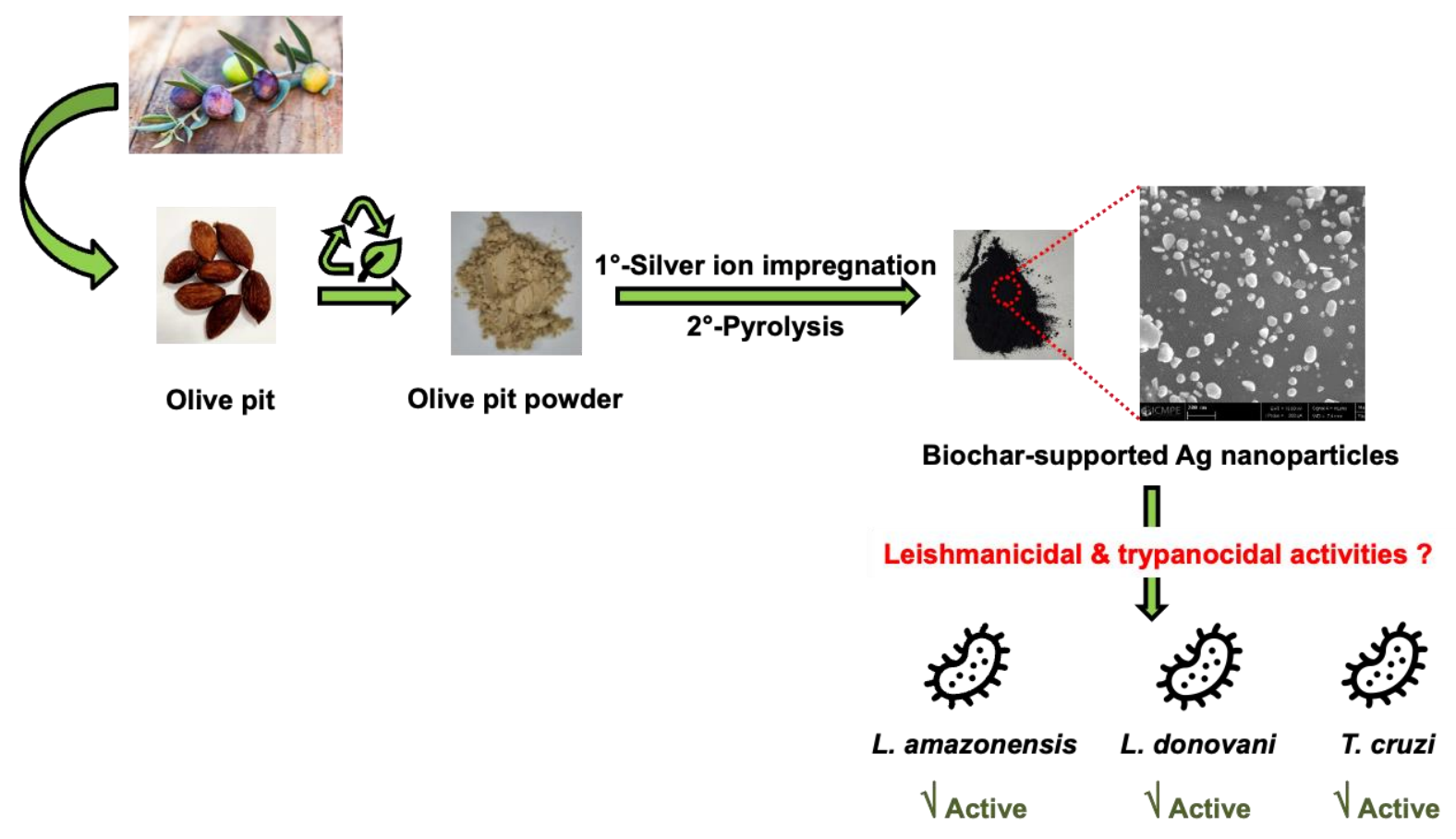




\section{Research Highlights}

$>$ Olive pit-derived biochar bearing silver nanoparticles has been prepared by one step slow pyrolysis

Silver nanoparticles are densely and evenly dispersed over biochar particles

$>$ Silver-loaded biochar has antiparasitic activity against L. donovani, L. amazonensis and T.cruzi.

$>$ Waste and junk conversion to bioactive nanocomposite opens new horizons in treating neglected tropical diseases. 


\section{Introduction}

Neglected Parasitic infection caused by kinetoplastids, a group of flagellated protozoans including Leishmania and Trypanosoma species, affect millions of persons and are responsible for high mortality, disability and morbidity rates [1, 2]. Leishmaniasis is considered as one of the major protozoan disease-causing high morbidities throughout the world. This infection is caused by a protist parasite of the genus Leishmania through the bite of a female phlebotomine sand fly [3]. Based on its clinical symptoms Leishmaniasis is classified in 3 different forms namely cutaneous, mucocutaneous and visceral leishmaniasis. As for Chagas disease (American trypanosomiasis), is caused by a protist parasite of the species Trypanosoma cruzi (T. cruzi) and is transmitted by the faeces of an hematophagous insects of the subfamily Triatominae [3]. This disease is classified into acute and chronic phases [4]. According to World Health Organization (WHO) 6 to 7 million people worldwide are estimated to be infected with Trypanosoma cruzi [5]. The actual treatment for those diseases is characterized by variable efficacy among different species, high toxicity and high cost resulting the urgent need for alternative anti-kinetoplastid agents. Metallic Silver (Ag), ions and their composites and compounds are well known to exhibit antiviral, antileishmanial and antibacterial activity [6]. There are numerous examples in the literature for antileishmanial applications using Ag such as the use of Chitosan-based Ag nanoparticles [7], $\mathrm{Au}_{\text {core }} @ \mathrm{Ag}_{\text {shell }}$ nanoparticles [8], meglumine antımoniate- $\mathrm{TiO}_{2} @ \mathrm{Ag}$ nanoparticle [9], goldsilver bimetallic nanoparticles [10] and Ag compound with imidazolidine-2-thione [11], to name but a few. Comparative studies using different NPs have found the following antileishmanial activity: $\mathrm{Ag}>\mathrm{Au}>\mathrm{TiO}_{2}>\mathrm{ZnO}>\mathrm{MgO}$. Further, their activity is enhanced by infrared and UV light [12]. Ag NPs produced with the help of olive and fig extract are more efficient than standard drug Pentostam [13].

There are limited reports on carbonaceous based materials for anti-kinetoplastid application and out of which they are mostly utilising functionalized carbon nanotubes (CNT), graphene and CNT-graphene [14]. In the context of "trash to treasure" or "waste to wealth", there has been an effort to synthesize Ag NPs with nanoxylan (xylan from corn cobs as a stabilizing and reducing agent) for antifungal and antileishmanial application [15]. In order to overcome the problem of silver resistance [16] and for biomass waste valorization purpose, biochar ("green carbon") having biocompatibility [17] is a perfect candidate in association with Ag NPs for antileishmanial applications. To the best of our knowledge, there are no reports on biochar with loaded silver nanoparticles for such application. 
Herein we report on novel silver-loaded biochar (Biochar@Ag) composite prepared by slow pyrolysis of olive stone agrowaste powder impregnated with silver nitrate. The resulting Biochar@Ag was characterized by XRD, SEM/EDX, TGA and XPS and its antiparasitic activity was evaluated against promastigotes stage of Leishmania donovani, Leishmani amazonensis and epimastigotes of Trypanosoma cruzi. The cytotoxicity towards host cells, murine macrophage, was also studied. All assays were conducted in vitro using the alamarBlue ${ }^{\circledR}$ method.

\section{Experimental}

\subsection{Fabrication of Biochar@Ag by slow pyrolysis}

Olive stone powder (3.67 g) was impregnated with an ethylic solution of silver nitrate (Aldrich, $584 \mathrm{mg}, \sim 3.44 \mathrm{mmol}$ in $10 \mathrm{~mL}$ ethanol) and left to dry. The powder was mixed from time to time on a glass lens and dried at RT until no more change in weight of the uimpregnated powder was noted. $1.2 \mathrm{~g}$ of AgNO3-impregnated olive stone powder was pyrolyzed in a tubular furnace (Thermolyne, model 21100) at $400{ }^{\circ} \mathrm{C}$ for $15 \mathrm{~min}$, under a stream of $\mathrm{N}_{2} / \mathrm{H}_{2}(95 / 5 \%)$ mixture. The temperature ramp was $30^{\circ} \mathrm{C} / \mathrm{min}$, i.e. slow pyrolysis regime. The system was left to cool under $\mathrm{N}_{2} / \mathrm{H}_{2}$ mixture. The Biochar@ Ag weight was found to be $\sim 0.44 \mathrm{~g}$ corresponding to a yield of $\sim 37 \%$.

The same agrowaste, without any silver nitrate, was pyrolyzed and the pyrolysis yield was found to be $\sim 26 \%$.

\subsection{Characterization of Biochar materials}

X-ray diffraction (XRD) was used for structural characterization using a D8 Advance Bruker diffractometer $\left(\mathrm{Cu} \mathrm{K} \alpha\right.$ radiation). Data were acquired in the $5-80^{\circ} 2 \theta$ range (step size $=0.01$ $\left.{ }^{\circ}\right)$ using an incident wavelength $\lambda$ of $1.54056 \AA$.

XPS analysis of Biochar@Ag was performed using a NEXSA apparatus (Thermo), fitted with monochromated X-ray beam and operated in the constant analyzer energy mode. The pass energy was set to 200 and $80 \mathrm{eV}$ for the survey and narrow regions, respectively. A flood gun was employed to compensate for the static charge buildup.

SEM images and EDX spectra were obtained with a Zeiss Merlin Field Emission scanning electron microscope (Oberkochen, Germany), operated at $5 \mathrm{kV}$ and coupled with a SDD XMax from Oxford Instruments. The biochar samples were coated with a $3 \mathrm{~nm}$-thin layer of 
palladium in order to avoid static charge. Palladium was deposited using a Cressington 208HR sputter-coater coupled with a Cressington MTM-20 thickness controller.

Thermogravimetric analyses were conducted using a Setaram machine (Setsys Evolution model). The samples were heated up from RT to $800{ }^{\circ} \mathrm{C}$, in air, at a heat rate of $10{ }^{\circ} \mathrm{C} / \mathrm{min}$.

2.3. In vitro Leishmanicidal and trypanocidal assays of Biochar@ Ag

\subsubsection{Parasite strains}

The anti-kinetoplastid activity of Biochar and Biochar@Ag was evaluated against the promastigote stage of Leishmania donovani (MHOM/IN/90/GE1F8R) and Leishmania amazonensis (MHOM/BR/77/LTB0016) and epimastigote form of Trypanosoma cruzi (Y strain).

\subsubsection{In vitro leishmanicidal effect}

The in vitro assay was performed using the alamarBlue ${ }^{\circledR}$ method as previously described (paper atteneri). This simple and rapid test is based on the reduction of non-fluorescent rezasurin into fluorescent resorufin by viable cells. This reaction could be measured by colorimetric or fluorometric sensor. Both tested nanoparticle's Biochar and Biochar@Ag were resuspended in physiologic water at a suitable concentration. In Furter serial dilutions using Leishmania medium (RPMI 1640) were made in a sterilized 96-wellmicrotiter plates (Corning ${ }^{\mathrm{TM}}$ ) to get a final volume of $100 \mu \mathrm{L}$ per well. Later, $100 \mu \mathrm{L}$ of Leishmania donovani or Leishmania amazonensis were added to each well, at a concentration of $2 * 10^{6}$ cells $/ \mathrm{mL}$. Finally, $20 \mu \mathrm{L}$ of alamarBlue ${ }^{\circledR}$ reactant were added to the entire plate. After an incubation of $72 \mathrm{~h}$, the plate was checked up visually using an inverted microscope and the emitted fluorescence was measured with an EnSpire ${ }^{\circledR}$ Multimode Plate Reader (Perkin Elmer, Madrid, Spain) at $570 / 585 \mathrm{~nm}$. Leishmanicidal activity was expressed as $\mathrm{IC}_{50}$ values (the concentration of a sample which caused a $50 \%$ reduction in parasite viability). Those values were calculated by non-linear regression analysis.

\subsubsection{In vitro evaluation against epimastigotes of Trypanosoma cruzi}

The nanoparticles were tested against the epimastigotes of T. cruzi. Briefly, in 96-well plates samples resuspend in physiological water were serially diluted in $100 \mu \mathrm{L}$ of LIT medium supplemented with $10 \%$ heat-inactivated fetal bovine serum. In all tests. Later, epimastigotes 
in logarithmic growth phase were counted using the Invitrogen Tali Image-based Cytometer, adjusted to $10^{6}$ cells $/ \mathrm{mL}$, and $100 \mu \mathrm{L}$ were added to the 96-well plate. Finally, $20 \mu \mathrm{L}$ of alamarBlue ${ }^{\circledR}$ reactive were added to the entire plate to be incubated at $27^{\circ} \mathrm{C}$ for $72 \mathrm{~h}$. The plate was observed under an inverted microscope after $72 \mathrm{~h}$ of incubation and analyzed statistically as described in the leishmanicidal test.

\subsection{Cytotoxicity Assay}

The cytotoxicity assay was performed as described in literature [3]. Briefly, $2 \times 10^{5}$ of macrophages cells were seeded in a 96 -well plate in a $5 \% \mathrm{CO}_{2}$ incubator at $37^{\circ} \mathrm{C}$. Cells were allowed to adhere in $20 \mathrm{~min}$. Meanwhile, serial dilutions of the Biochar@Ag were prepared in a sterile deep well plate; later $50 \mu \mathrm{L}$ of each concentration were added to the macrophage 96 well plate. Finally, the alamarBlue ${ }^{\circledR}$ Reagent was added into each well at an amount equal to $10 \%$ of the medium volume and the plate was incubated for 24 hours at $37^{\circ} \mathrm{C}$ with $5 \% \mathrm{CO}_{2}$ atmosphere. The percentage of cell viability was evaluated using the alamarBlue ${ }^{\circledR}$ assay. Dose response curves were plotted and the $\mathrm{CC}_{50}$ values were calculated.

Statistical analysis: all data are expressed as the mean \pm standard deviation of at least three independent ex-periments. To highlight the dose response effect of the Biochar@Ag, a statistical comparison was conducted using one-way analysis of variance (ANOVA). All analyses and graphics were done by GraphPad Prism version 9.0. Statistical significance was set at $p<0.05$.

\section{Results and Discussion}

\subsection{Strategy of Biochar@ Ag design and antiparasitic application}

There are numerous ways to prepare metallic nanoparticle-loaded biochar. The direct method consists in impregnating the biomass precursor with silver nitrate followed by pyrolysis. The indirect method, consists in making biochar first then react with metal ions for the in situ deposition of the latter and their subsequent reduction into biochar-supported metallic nanoparticles. Of relevance to this work, Huang et al. [18] treated silver nitrate with $\mathrm{KOH}$ to obtain $\mathrm{AgO}$, and added rosin. The mixture was pyrolyzed at $400{ }^{\circ} \mathrm{C}$ for 8 min under air. In this work, we avoid the use of $\mathrm{KOH}$ or any acid treatment or post-treatment of the biochar. We have pyrolyzed silver nitrate-impregnated olive stone powder particles in the slow pyrolysis regime, at $400{ }^{\circ} \mathrm{C}$ for $15 \mathrm{~min}$ and the resulting material was tested for antiparasitic activity in comparison to bare biochar. Figure 1 depicts the whole strategy of the work. 


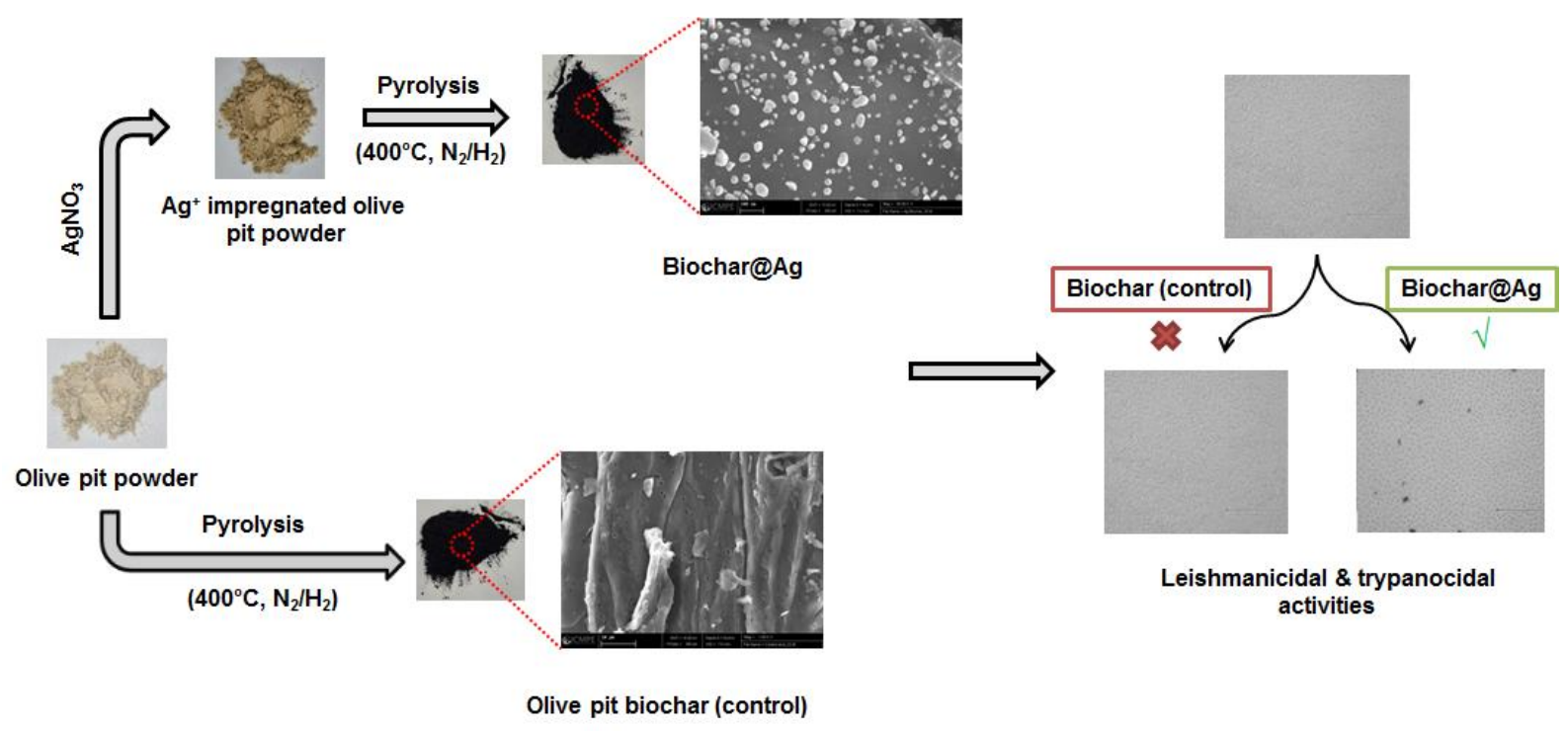

Figure 1. General procedure for making silver nanoparticle-loaded biochar for antikinetoplastid application.

\subsection{Physicochemical properties of Biochar and Biochar@Ag}

Figure 2 displays the XRD patterns of Biochar@Ag and the reference bare Biochar. The latter is amorphous and exhibits no diffraction peaks. In contrast, sharp diffraction peaks are noted for Biochar@Ag; they are centred at $2 \theta=37.9,44.0,64.3$ and $77.2^{\circ}$ and assigned to $\operatorname{Ag}(111), \operatorname{Ag}(200), \operatorname{Ag}(220)$ and $\operatorname{Ag}(311)$ crystallographic planes, respectively. This is in line with results reported elsewhere for silver NP-loaded rosin biochar prepared carbonization of rosin modified with $\mathrm{KOH}$-treated silver nitrate [18]. The XRD pattern of Biochar@Ag is consistent with pure metallic silver nanoparticles loaded on biochar.

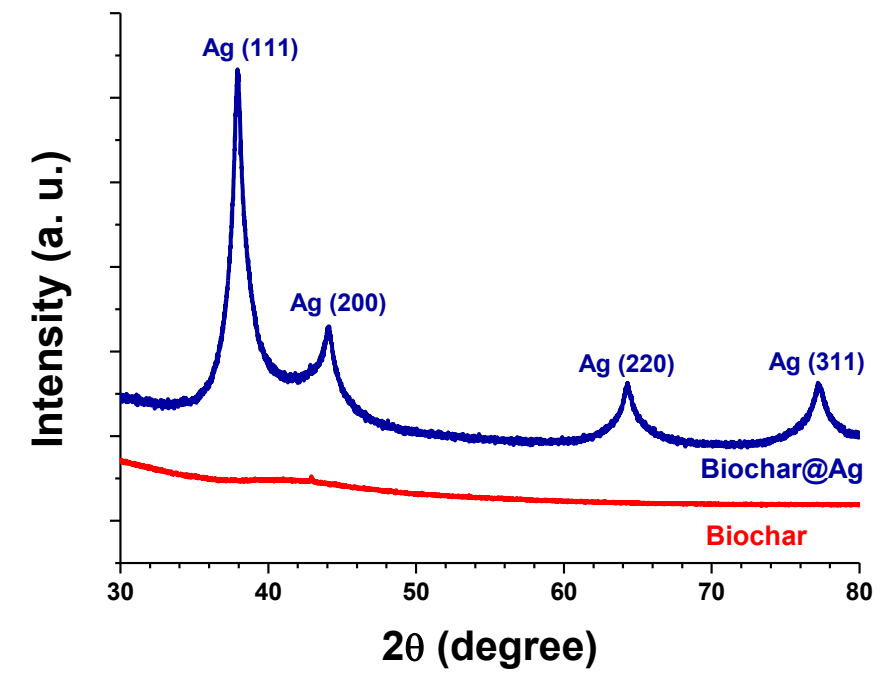

Figure 2. XRD patterns of bare Biochar and Biochar@Ag composite. 
XP spectra and surface elemental composition of Biochar@Ag are displayed in Figure 3. The survey region (Figure $3 \mathrm{a}$ ) exhibits sharp $\mathrm{Ag} 3 \mathrm{~d}$ doublet is noted with $\mathrm{Ag} 3 \mathrm{~d}_{5 / 2}$ and $\mathrm{Ag} 3 \mathrm{~d}_{3 / 2}$ peaks centred at 368.2 and $374.2 \mathrm{eV}$, respectively (Figure $3 \mathrm{~b}$ ). These binding energy values [19] and $6 \mathrm{eV}$ splitting [20] are consistent with silver in the metallic state. C1s narrow region from the biochar is displayed in Figure 3c; it is peak fitted with four components assigned to $\mathrm{sp}^{2}$ carbon atoms $(284.6 \mathrm{eV}), \mathrm{sp}^{3}$ carbon atoms $(285.3 \mathrm{eV}), \mathrm{C}-\mathrm{O}(286.8 \mathrm{eV})$ and $\mathrm{O}-\mathrm{C}=\mathrm{O}$ $(288.6 \mathrm{eV})$ carbon atom types [21]. Figure $3 \mathrm{~d}$ depicts the surface elemental composition (in at. \%); $\mathrm{O} / \mathrm{C}$ ratio is about 0.16 , lower than 0.20 obtained for Shorea robusta leaves, carbonized at $300{ }^{\circ} \mathrm{C}$ [20]. A lower pyrolysis temperature induces less degradation of the cellulosic initial material and thus leads to higher $\mathrm{O} / \mathrm{C}$ ratio as is the case in the case of $S$. robusta [20]. One interesting point is that the actual surface silver content is high, and parallels the sharp Ag3d doublet noticed in Figure 3a. This indicates that direct pyrolysis of silver nitrate-impregnated biomass is an effective way to obtain high silver nanoparticle loading on biochar, compared to the impregnation of biochar with pre-fabricated silver nanoparticles. Indeed, the survey region reported by Shaikh et al. [20] exhibits a very low relative intensity of the Ag3d doublet. 


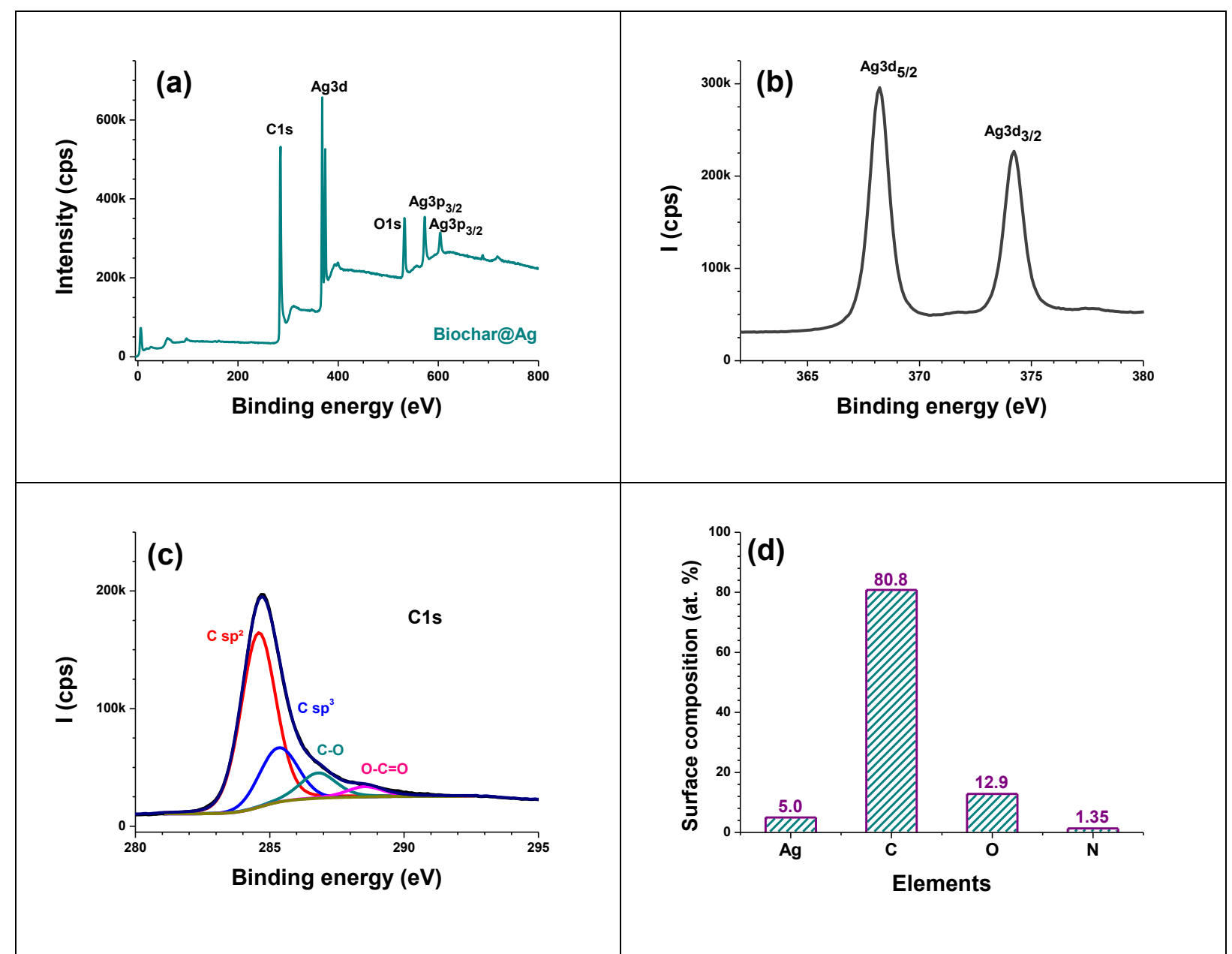

Figure 3. XP spectra and composition of Biochar@ Ag: (a) survey, (b) Ag3d, (c) C1s, and (d) chemical composition in at.\%.

SEM/EDX can further give credit to the XPS analysis and could explain the sharp Ag3d peak. Figure 4 displays SEM/EDX analyses of Biochar and Biochar@Ag. One can clearly note that very dense distribution of silver nanoparticles (Figures $4 \mathrm{~d}, \mathrm{e}$ ) over the biochar microparticles, which have almost a wax-shaped structure (Figure 4a,b). No particular feature is noted for Biochar at higher magnification (Figure 4c), which contrasts with the individual or stacked, triangular silver nanoparticles $(68 \pm 7 \mathrm{~nm})$ displayed in Figure $4 \mathrm{~g}$ for Biochar@ Ag. It is also worth to note the uniform distribution of the Ag NPs (Figure 4f), which fully credits the effective simple impregnation approach devised in this work. Prominent Ag peak is noted in Figure 4h for Biochar@Ag compared to Figure 4d showing te absence of any Ag peak for the bare Biochar. The $\mathrm{Ag} / \mathrm{C}$ ratio is $~ 0.04$ and $\sim 0.06$ as determined by EDX (Figure $4 \mathrm{~h}$ ) and by XPS, respectively. This is an expected trend as XPS is more surface specific than EDX. 

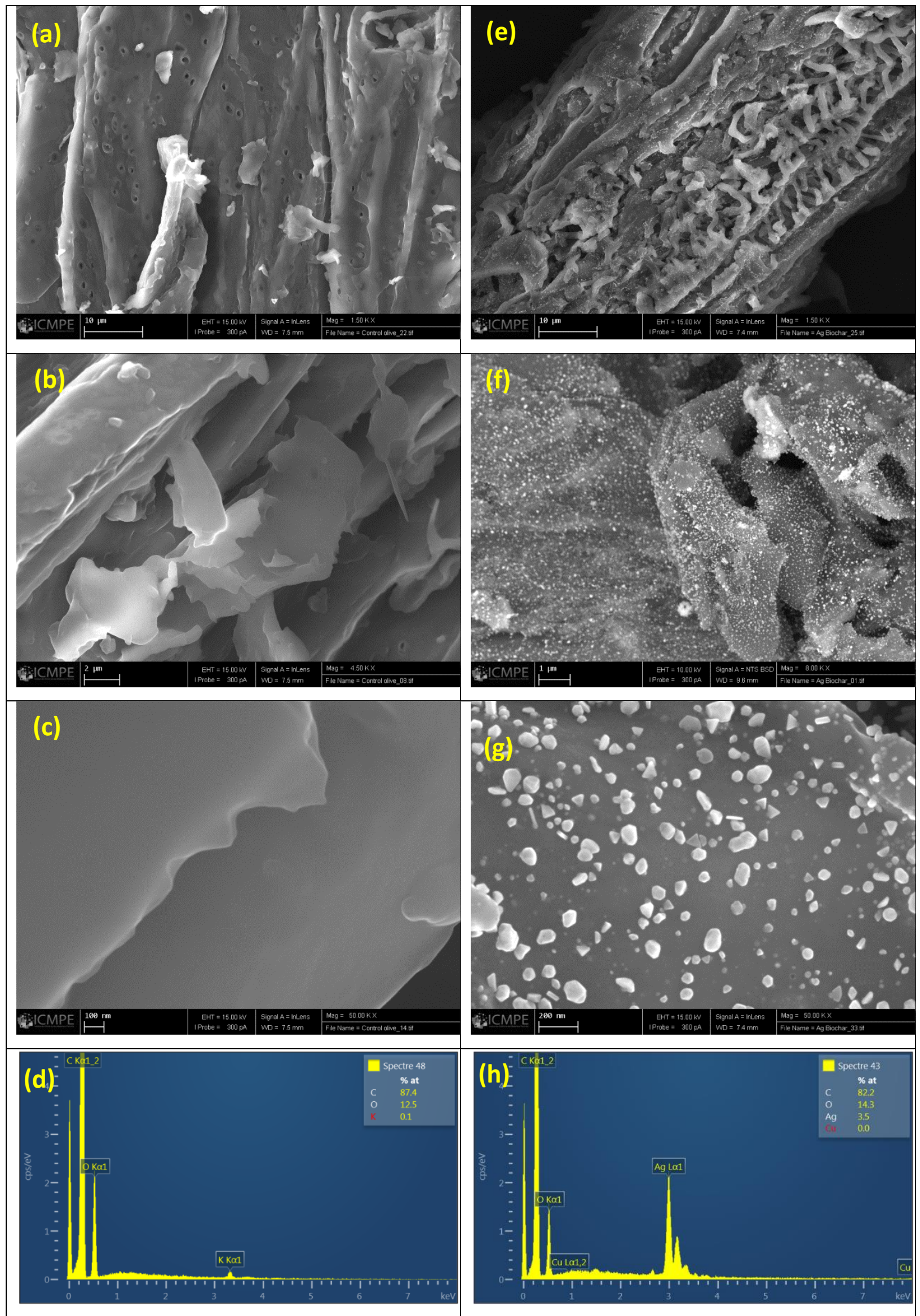

Figure 4. SEM (a-c, e-g) and EDX (d,h) analyses of Biochar (a-d) and Biochar@ Ag (e-h) at various magnification. EDX spectra in $\mathrm{d}$ and $\mathrm{h}$ are representative of all EDX analyses. 


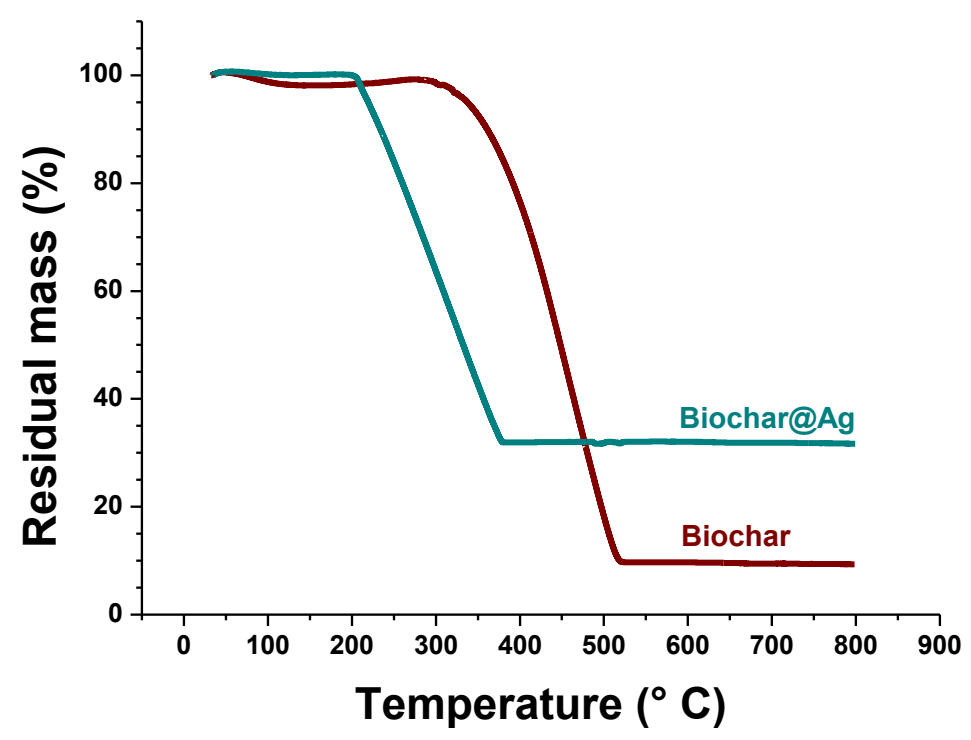

Figure 5. TGA analyses of Biochar and Biochar@ Ag.

Figure 5 plots the thermograms of Biochar@Ag and the reference bare Biochar. The gravimetric measurements were performed under air in order to burn out the biochar; this is the reason for low residual 9.7 wt. \%. In the case of Biochar@ Ag, the higher residual mass (31.5\%). The onset of Biochar degradation is $274{ }^{\circ} \mathrm{C}$, higher than that of Biochar@ Ag which starts to degrade at $200{ }^{\circ} \mathrm{C}$, well below the bare Biochar. This could be due to catalytic thermal degradation of the carbonized matter by silver NPs [22]. Degradation progresses until $377{ }^{\circ} \mathrm{C}$ and the mass becomes stable. In the case of Biochar, thermal degradation keeps progressing until reaching $522{ }^{\circ} \mathrm{C}$ the onset of stable Biochar weight loss.

\subsection{Antikinetoplastid activities}

Table 1. Summary of $\mathrm{IC}_{50}$ and $\mathrm{CC}_{50}$ values obtained in vitro

\begin{tabular}{lcccc}
\hline & $\begin{array}{c}\text { Trypanosoma cruzi } \\
\mathbf{I C}_{\mathbf{5 0}}\end{array}$ & $\begin{array}{c}\text { Leishmania } \\
\text { donovani } \\
\mathbf{I C}_{\mathbf{5 0}}\end{array}$ & $\begin{array}{c}\text { Leishmania } \\
\text { amazonensis } \\
\text { IC }_{\mathbf{5 0}}\end{array}$ & $\begin{array}{c}\text { Murine } \\
\text { Macophages } \\
\mathbf{C C}_{\mathbf{5 0}}\end{array}$ \\
\hline Biochar@ Ag $(\mathbf{p p m})$ & $12.154 \pm 0.206^{\mathrm{b}}$ & $9.942 \pm 0.900^{\mathrm{a}}$ & $14.555 \pm 1.035^{\mathrm{c}}$ & $70.33 \pm 0.550$ \\
\hline Benznidazole $(\boldsymbol{\mu M})$ & $6.940 \pm 1.940$ & $\mathrm{NT}$ & $\mathrm{NT}$ & $>400 \mu \mathrm{M}$ \\
\hline Miltefosine $(\boldsymbol{\mu M})$ & $\mathrm{NT}$ & $3.32 \pm 0.27$ & $6.48 \pm 0.24$ & $72.190 \pm 3.060$ \\
\hline$*$ Means within strains with different lower-case letters $(\mathrm{a}-\mathrm{c})$ are significantly different $(p<0.05)$.
\end{tabular}



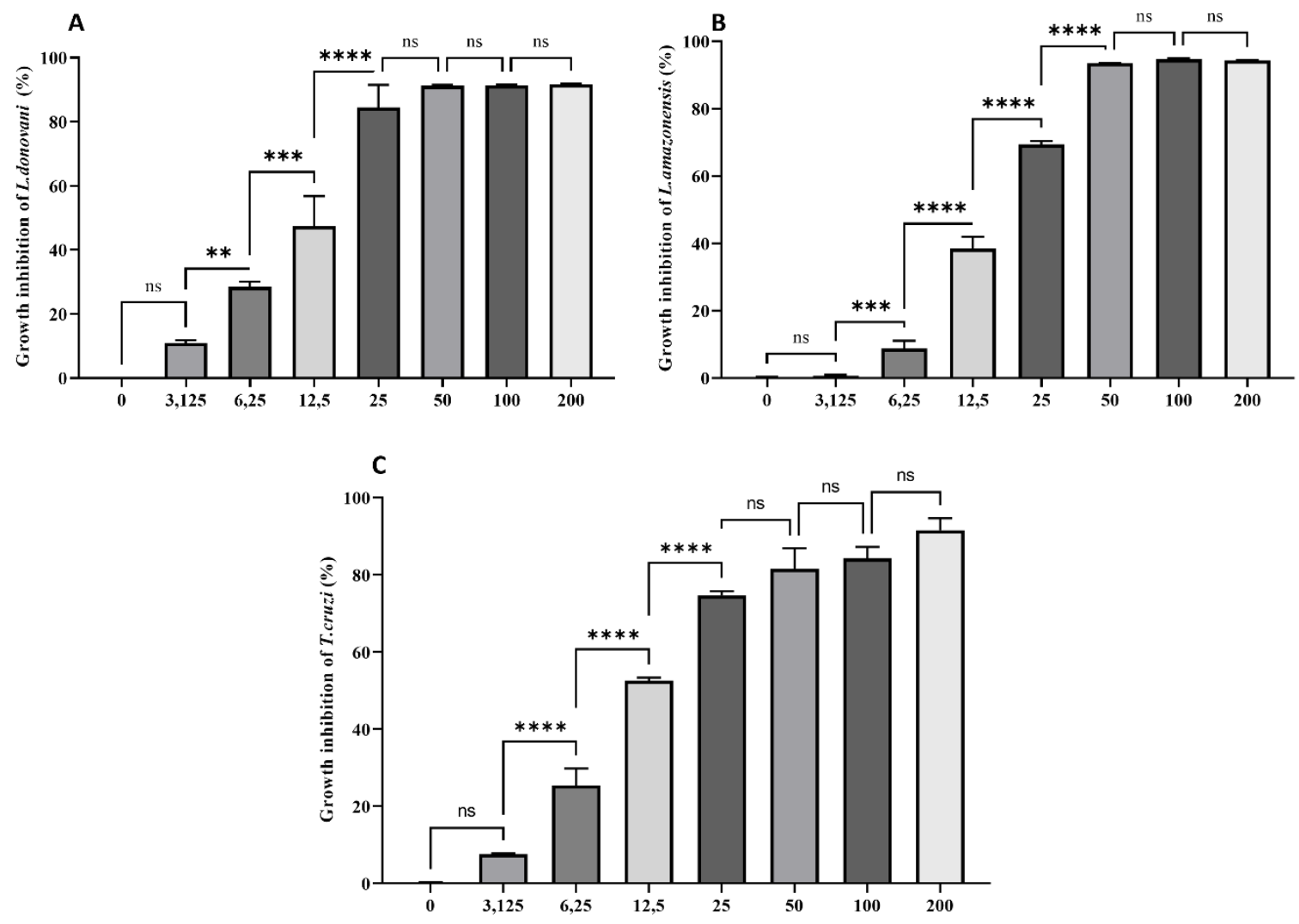

Figure 6. Effect of the Biochar@Ag on promastigote stage of $L$. donovani (A), $L$. amazonensis (B) and Trypanosoma cruzi (C). The bar graph includes the calculated percentage of growth inhibition. Differences between the values were assessed using one-way analysis of variance (ANOVA). Data are presented as means $\pm \mathrm{SD}(\mathrm{N}=3) * * p<0.01$; $* * * p<$ $0.001 ; * * * * p<0.0001$.

Leishmanicidal and trypanocidal activities of Biochar and Biochar@Ag were determined using almarBlue ${ }^{\circledR}$ method. The assay was done against promastigotes stage of $L$. amazonensis and L. donovani and epimastigotes of $T$. cruzi. The obtained values of concentrations inhibiting $50 \%$ of the parasite $\left(\mathrm{IC}_{50}\right)$ are summarized in Table 1 . The antiprotozoal activity was dose dependent as illustrated in the Figure 6. The natural Biochar was inactive against all the tested strains. In contrast, Biochar@Ag was very effective to inhibit all the strains with $\mathrm{IC}_{50}$ of $12.154 \pm 0.206,9,942 \pm 0.900$ and $14.555 \pm 1.035 \mathrm{ppm}$ against T.cruzi, L. dononvani and L. amazonensis, respectively. The ANOVA analysis revealed that the antikinetoplastid capacity was statistically affected by the dose and the type of parasite used. Indeed, the most sensitive parasite was L. donovani where a concentration of 25 ppm was able to inhibit $90 \%$ of the initial cell culture (Figure 7C and 7D), whereas a concentration of $50 \mathrm{ppm}$ was needed to inhibit $90 \%$ of Trypanosoma cruzi (7E and 7F) and Leishmania amazonensis (Figure 7A, B). On the other hand, the toxicity of the nanoparticles 
was evaluated towards murine macrophages and the cytotoxic concentration at $50 \%$ was determined. The $\mathrm{CC}_{50}$ of the Biochar@Ag was $70.33 \pm 0.550$.

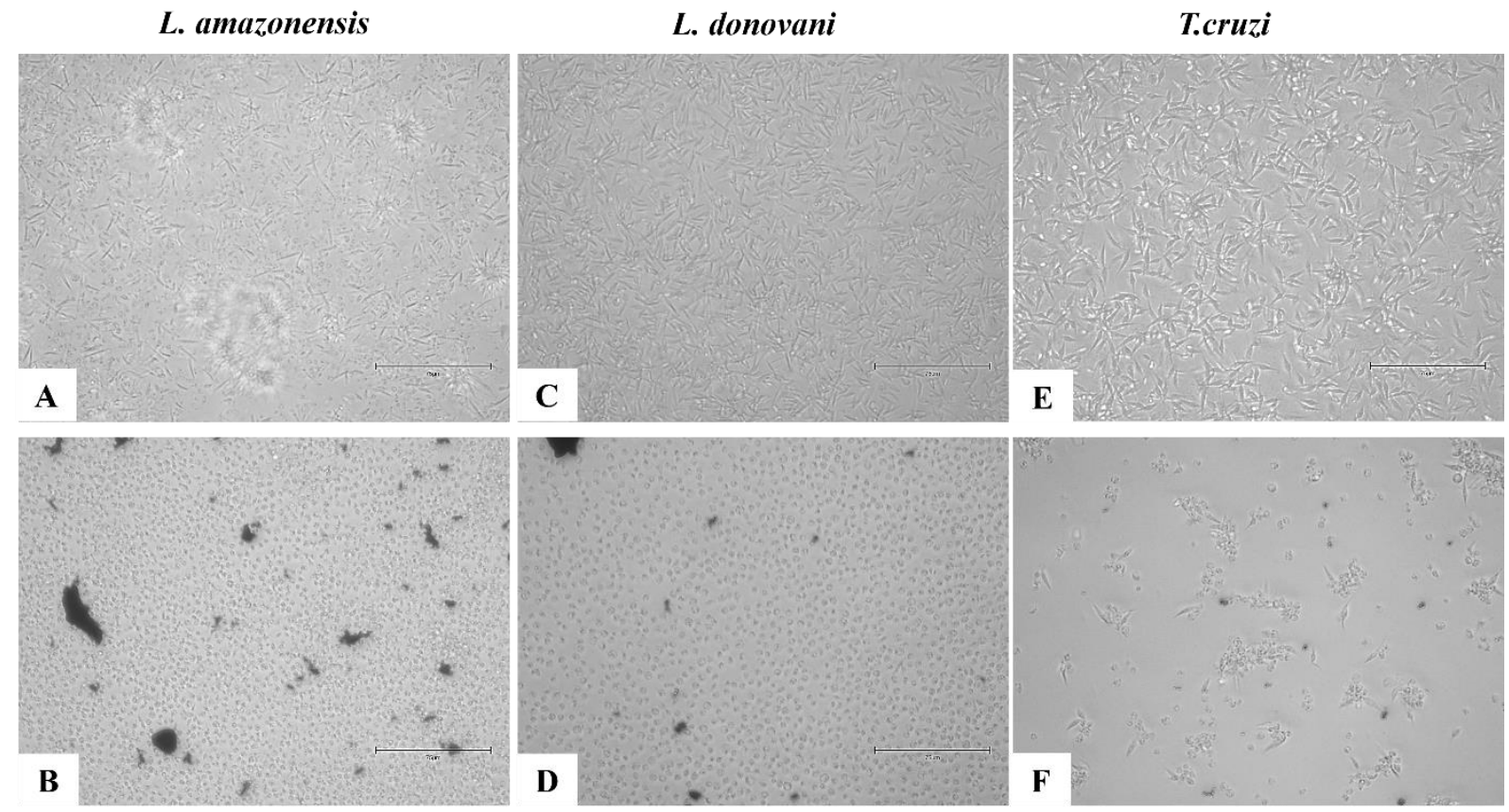

Figure 7. Effect of Biochar@Ag on Leishmania amazonensis at 25ppm; Leishmania donovani at $12.5 \mathrm{ppm}$ (D) and Trypanosoma cruzi at $25 \mathrm{ppm}$ (F). Negative control for each strains (A, C and E). Images (40X) are representative of the cell population observed in the performed experiments. Images were obtained using an EVOS FL Cell Imaging System AMF4300, Life Technologies, USA. Scale bar $=75 \mu \mathrm{m}$.

\section{Conclusion}

To sum up, we have prepared silver nanoparticle-loaded biochar (Biochar@ Ag) by pyrolysis of silver nitrate -impregnated olive pit powder at $400{ }^{\circ} \mathrm{C}$ for $15 \mathrm{~min}$, in the slow regime (heat rate $=30^{\circ} \mathrm{C} / \mathrm{min}$ ). Dense and even distribution of triangular silver NPs was noted by SEM, which accounts for very sharp Ag3d peaks recorded by XPS surface analysis. A more porous structure was observed for Biochar@Ag compared to bare Biochar, possibly implying an effect of silver nanoparticles which catalyze the degradation or the biomass or alter its structure during pyrolysis. A concentration of 50 ppm of Biochar@ Ag was able to eliminate $90 \%$ of three different kinetoplastid including Leishmania donovani, causative organism of kala-azar and visceral leishmaniasis, Leishmania amazonensis, causative agent of cutaneous leishmaniasis, and Trypanosoma cruzi, causative agent of Chagas disease.

From what has been stated above, we have provided a simple pyrolysis method, in the slow regime, to design antiparasitic application of Biochar@Ag against $L$. donovani, $L$. amazonensis and T.cruzi with lower cytotoxic effect on murine macrophage; although further studies are needed to illustrate its action mode on the parasite. This waste-to-wealth procedure could be adapted for other anti-tropical parasite applications, and more importantly it could be scaled up given its simplicity, versatility and low-cost. 


\section{Acknowledgements}

The authors would like to thank both the French and Egyptian Governments for funding AMK's contribution through a fellowship granted by the French Embassy in Egypt (Institut Francais d'Egypte) and Science and Technology Development Fund (STDF)-Egypt, Project number (42248). Wallonie Bruxelles International (WBI) is acknowledged for the provision of a grant "Bourse WBI Excellence World" (No Imputation 101386, Article Budgétaire 33.01.00.07). This study was supported as well by the RICET (project no. RD16/0027/0001 of the programme of Redes Temáticas de Investigación Cooperativa, FIS), CIBER de Enfermedades Infecciosas (CIBERINFEC), Instituto de Salud Carlos III (CB21/13/00100) and Cabildo de Tenerife 21/0587 cofunded by FDCAN and MEDI (Tenerife Innova Programme).

\section{References}

1. Stuart, K., et al., Kinetoplastids: related protozoan pathogens, different diseases. The Journal of clinical investigation, 2008. 118(4): p. 1301-1310.

2. Lin, C., et al., N6-modification of 7-Deazapurine nucleoside analogues as Anti-Trypanosoma cruzi and anti-Leishmania agents: Structure-activity relationship exploration and In vivo evaluation. European Journal of Medicinal Chemistry, 2022: p. 114165.

3. de Fuentes-Vicente, J.A., et al., What makes an effective Chagas disease vector? Factors underlying Trypanosoma cruzi-triatomine interactions. Acta Tropica, 2018. 183: p. 23-31.

4. Villalta, F., et al., VNI cures acute and chronic experimental Chagas disease. The Journal of infectious diseases, 2013. 208(3): p. 504-511.

5. Rassi, A. and J.M. de Rezende, American trypanosomiasis (Chagas disease). Infectious Disease Clinics, 2012. 26(2): p. 275-291.

6. Allahverdiyev, A.M., et al., Antileishmanial effect of silver nanoparticles and their enhanced antiparasitic activity under ultraviolet light. International journal of Nanomedicine, 2011. 6: p. 2705.

7. Lima, D.d.S., et al., Chitosan-based silver nanoparticles: A study of the antibacterial, antileishmanial and cytotoxic effects. Journal of Bioactive and Compatible Polymers, 2017. 32(4): p. 397-410.

8. Ghosh, S., et al., Dioscorea bulbifera mediated synthesis of novel AucoreAgshell nanoparticles with potent antibiofilm and antileishmanial activity. Journal of Nanomaterials, 2015. 2015.

9. Abamor, E.S., et al., Meglumine antımoniate-TiO2@ Ag nanoparticle combinations reduce toxicity of the drug while enhancing its antileishmanial effect. Acta tropica, 2017. 169: p. 3042.

10. Alti, D., et al., Gold-Silver Bimetallic Nanoparticles Reduced with Herbal Leaf Extracts Induce ROS-Mediated Death in Both Promastigote and Amastigote Stages of Leishmania donovani. ACS omega, 2020. 5(26): p. 16238-16245.

11. Espuri, P.F., et al., Synthesis and evaluation of the antileishmanial activity of silver compounds containing imidazolidine-2-thione. JBIC Journal of Biological Inorganic Chemistry, 2019. 24(3): p. 419-432.

12. Jebali, A. and B. Kazemi, Nano-based antileishmanial agents: a toxicological study on nanoparticles for future treatment of cutaneous leishmaniasis. Toxicology in vitro, 2013. 27(6): p. 1896-1904. 
13. Almayouf, M.A., et al., The effects of silver nanoparticles biosynthesized using fig and olive extracts on cutaneous leishmaniasis-induced inflammation in female balb/c mice. Bioscience Reports, 2020. 40(12): p. BSR20202672.

14. Gedda, M.R., et al., Evaluation of safety and Antileishmanial efficacy of amine functionalized carbon-based composite nanoparticle appended with amphotericin B: An in vitro and preclinical study. Frontiers in chemistry, 2020. 8: p. 510.

15. Silva Viana, R.L., et al., Green synthesis of antileishmanial and antifungal silver nanoparticles using corn cob xylan as a reducing and stabilizing agent. Biomolecules, 2020. 10(9): p. 1235.

16. Chopra, I., The increasing use of silver-based products as antimicrobial agents: a useful development or a cause for concern? Journal of antimicrobial Chemotherapy, 2007. 59(4): p. 587-590.

17. Yıldızlı, G., G. Coral, and F. Ayaz, Biochar as a Biocompatible Mild Anti-Inflammatory Supplement for Animal Feed and Agricultural Fields. Chemistry \& biodiversity, 2021.

18. Huang, J.-F., et al., Facile pyrolysis preparation of rosin-derived biochar for supporting silver nanoparticles with antibacterial activity. Composites Science and Technology, 2017. 145: p. 89-95.

19. Abbas, Q., et al., Biochar-induced immobilization and transformation of silver-nanoparticles affect growth, intracellular-radicles generation and nutrients assimilation by reducing oxidative stress in maize. Journal of hazardous materials, 2020. 390: p. 121976.

20. Shaikh, W.A., R.U. Islam, and S. Chakraborty, Stable silver nanoparticle doped mesoporous biochar-based nanocomposite for efficient removal of toxic dyes. Journal of environmental chemical engineering, 2021. 9(1): p. 104982.

21. Kim, D.-G. and S.-O. Ko, Effects of thermal modification of a biochar on persulfate activation and mechanisms of catalytic degradation of a pharmaceutical. Chemical Engineering Journal, 2020. 399: p. 125377.

22. Giudicianni, P., et al., Inherent metal elements in biomass pyrolysis: A review. Energy \& Fuels, 2021. 35(7): p. 5407-5478. 